Proceedings of SALT 26: 601-619, 2016

\title{
Presuppositions and salience: An experimental approach *
}

\author{
Jacques Jayez \\ ENS de Lyon and Institut de Sciences \\ Cognitives Marc Jeannerod, CNRS \\ Rob Reinecke \\ Institut de Sciences Cognitives Marc \\ Jeannerod, CNRS
}

\begin{abstract}
We present an EEG-based experimental investigation on additive discourse continuations of factive sentences according to a pattern: Paul knows that Peter takes the bus. Louis too takes/knows .... We want to determine whether reference to the main content (with knows) or to the presupposition (with takes) elicits a different brain response. We conclude from the data that there is no trace of electrical waveforms usually associated with deviation from a norm or reprocessing, although there is an observable moderate contrast in the $250-400 \mathrm{~ms}$ time window at frontal sites, which is in need of controlled replication to be properly interpreted.
\end{abstract}

Keywords: presupposition, salience, at issue content, experimental linguistics, ERP, N400, P600

\section{Introduction}

The literature on presuppositions has its fundamental raison d'être in a distinction between the main content (MC) and the presupposition (PP) conveyed by presuppositional expressions. Most observations converge towards the conclusion that it is difficult for a PP to be the focus of an utterance or to be a focus source, that is, to introduce a question that subsequent discourse is going to address. We take the distinction between PP and MC to be empirically well-established in this regard. However, the cognitive inner workings of the distinction are still largely unexplored and the role of PPs as topics is not well-understood. In particular, it is not clear whether the asymmetry of MC and PP extends to the topic function, perhaps in virtue of an intrinsic salience difference between MC and PP. The goal of this paper is to present the first steps of an experimental approach to the question of PPs as topics. We use an EEG-based technique and an additive relation marked by aussi (too) in French in order to determine whether reference to the MC and to the PP elicits different brain responses. We first provide a general linguistic (Section 2.1) and experimental (2.2) background to motivate the two experiments described in

* We thank the reviewers for and audience at SALT 26, as well as our colleagues at the Institut de Sciences Cognitives for their helpful questions, comments and suggestions.

C2016 Jayez and Reinecke 
Jayez and Reinecke

Sections 3.1 and 3.2, before discussing the results in Section 3.3. We conclude by deriving from the results and the general state-of-the art some reasonable experimental follow-ups (Section 4).

\section{Motivation for the experiment}

\subsection{Some fundamental intuitions}

Although the literature on presuppositions is large, there are a number of recurring themes that suggest the existence of common intuitions. We will mention the three features which seem to be dominant across the works of the last forty years.

The first feature is that the expressions which convey a presupposition (the so called presupposition triggers) jointly communicate two pieces of information, the main content (MC) or at-issue content and the presupposition (PP). For instance, Mary stopped dancing communicates that Mary has not been dancing after some past time point $t$ (the MC) and that she was dancing before $t$ (the PP). According to Potts (2005), this twofold informational character contrasts with conventional implicatures, for instance expressives like damn, appositives or evaluative adverbs like unfortunately. In Unfortunately, Mary stopped dancing, the adverb communicates that the proposition/fact conveyed by the clause to which it adjoins is regrettable (presumably for the speaker). The proposition/fact evaluated by the adverb is in a sense independent from the implicature trigger (unfortunately), in that suppressing the trigger leaves the proposition/fact untouched.

The second feature corresponds to three types of observations, which can be grouped together under the general heading of 'discourse non-integration'.

Discourse connection PPs generally resist discourse connection (Ducrot 1972). (1) is an unproblematic sentence, which asserts that Paul has no caviar for breakfast because caviar is too expensive and presupposes that he had caviar before. The because-clause is interpreted as targeting the MC. In contrast, (2) is (more) difficult to interpret and, whatever construal a reader/hearer is able to come up with, ${ }^{1}$ it most probably won't be an attachment to the PP, that is, the sentence is not going to be interpreted as 'Paul does not have caviar for breakfast and he had caviar before because he liked caviar'.

(1) Paul stopped having caviar for his breakfast because it's too expensive.

(2) \# Paul stopped having caviar for his breakfast because he liked that.

1 A possible construal, in the context of (1), is that Paul wanted to save money and decided to stop caviar because he liked caviar so much that he spent too much money on it. 
Presuppositions and salience

Answerhood PPs do not in general provide natural answers to questions (Grimshaw 1979). The two responses in (3) sound off the track.

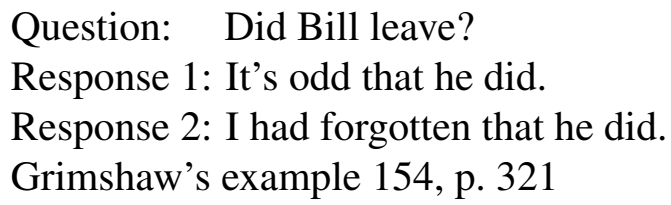

Projection Finally, there is the well-known projection phenomenon, the fact that PPs are not necessarily affected by truth-inversion (negation) or truth-suspension operators (interrogation, modals), which negate or suspend the MC. For instance, with Did Mary stop dancing?, the MC is not asserted whereas the PP is still there. See Beaver \& Geurts 2013; Chierchia \& McConnell-Ginet 1990; Geurts 1999 for more detailed lists of projection configuration.

The third and last feature is the backgrounded nature of PPs. Stalnaker (1974) defends the view that PPs are 'normally' members of the common ground when the PP trigger is used in a sentence. It is not easy to form a clear conception of what 'normally' could mean. In a famous passage, Stalnaker (1974: 473) introduces the idea that a speaker presupposes that $P$ if he believes or assumes that $P$, plus a number of other conditions. ${ }^{2}$ However, Stalnaker (1974: 474) concedes that sometimes there is no such belief or assumption: "In such a case, a speaker tells his auditor something in part by pretending that his auditor already knows it. The pretense need not be an attempt at deception. It might be tacitly recognized by everyone concerned that this is what is going on, and recognized that everyone else recognizes it."

This modulation of the main theme anticipates the recent Abbott vs. Stalnaker debate (Abbott 2008; Stalnaker 2008). In a nutshell, if PPs can be new information ('informative'), what room is left for their backgrounded character? Stalnaker had already faced this problem in Stalnaker 1974: 480, fn 3 when he discusses Sadock's example of an informative PP. B's answer is appropriate even in a context where it is clear that A did not know that B has a sister.

\section{A: Are you going to lunch?}

B: No, I've got to pick up my sister.

Examples such as (4) prompted Stalnaker to reconsider his definition of the common ground, essentially by separating the set of common beliefs from the common ground proper, which he defines as follows in Stalnaker 2002: 716.

\section{Common ground}

It is common ground that $\phi$ in a group if all members accept (for the purpose

2 These conditions have to do with shared beliefs and are not directly relevant to our discussion. 
of the conversation) that $\phi$, and all believe that all accept that $\phi$, and all believe that all believe that all accept that $\phi$, etc.

Acceptance corresponds to treating a proposition as true for some reason. In contrast to common ground as shared belief, the new notion of common ground allows for situations in which participants all doubt that $\phi$ is true while accepting it for various reasons and believing that they (believe that ... they believe that they) accept it, see the Martini example, inspired from Donellan, in Stalnaker 2002: 717718.

In this new approach to common ground, speaker presuppositions "will be, as before, the speaker's beliefs about the common ground" (Stalnaker 2002: 717). Clearly, there is a temporal dimension to be added to make the proposal more coherent. At the moment the corresponding PP trigger is used, a truly informative PP does not coincide with a belief that the PP is accepted. The option that Stalnaker seems to favor is one in which the speaker believes that the PP will be accepted at the end of the speech act ("at the appropriate point in the conversation" (Stalnaker 2002: 710)). This is in particular the case when the speaker is presumed to be competent, as in the case of the sister example (4).

However, it has been argued that the time-based account of Stalnaker might run into problems with sentences of type (6), see Szabó 2006; von Fintel 2006. Imagine a context in which it is common belief that some of the addressees don't know that the speaker has a sister. Intuitively, (6) might sound odd, even if we assume that the existence of a sister is accommodated after the speech act, given that the speaker is normally competent about the structure of her family. A straightforward explanation for the oddness of (6) is that, at the time the utterance is issued, it might be false that the existence of a sister is in the common ground, and so it would be simply wrong in that case to assert that the participants all know that the speaker has a sister. A way out is to assume that the present tense in (6) picks out a time before accommodation (this is apparently von Fintel's interpretation of the problem, as construed by Szabó (2006: 7) and also one of Stalnaker's interpretations in his reply to Abbott (2008)). Szabó (2006: 7) casts doubt on this possibility by asking what the reference of the present tense is. If the reference can be shifted to a time point after the accommodation has taken place, there is no reason why (6) should be odd.

We all know that I have a sister.

Stalnaker's answer to Szabó (and to Abbott, who endorses Szabó's objection) in his 2008 paper takes into account two possibilities. If the present tense refers to a state of affairs simultaneous with the utterance, then, clearly, the sentence is false in a context where the existence of a sister is not common belief. If the present tense can refer to a point after the end of the statement, Stalnaker claims that the resulting 
meaning is "we all will know, as soon as I finish talking, that I have a sister", which is a strangely indirect way of communicating that the speaker has a sister. So, under the first interpretation, the sentence is false and, under the second, it is odd.

Stalnaker's second interpretation is spurious. No natural dialogue based on it can be constructed. Imagine the exchange in (7). It sounds very strange. What could B's answer mean? Certainly not "Yes, as soon as you finished talking I knew/learned that you had a sister".

A: You know that I have a sister.

$\mathrm{B}$ : Yes, this is exactly what happened.

So, von Fintel's answer to Szabó's objection is basically correct: if there is any oddness with (6), it is simply due to the fact that sentence makes reference to a time where what it asserts (that the existence of a sister is in the set of shared beliefs) is false. To see what can happen after (6) has been uttered, the example has to be put in the more general context of conversational planning.

The role of conversational planning has been studied from different perspectives, for instance quite generally in a theory of action and communication in the BeliefDesire-Intention (BDI) model (Wooldridge 2000) or in the more specific setting of Question Under Discussion (QUD) models (Roberts 2012; Ginzburg 2012). While there are significant differences between these families of models, they agree on the idea that a conversational move (or its absence, as when an utterance is not explicitly rejected) reflects the goals and anticipations of a speaker. If a contrary-to-facts utterance is issued at some point in the discourse, it can be accepted if its relevance to other, more important moves, is judged very weak or negligible. If the central criterion is the relevance with respect to conversational planning, it may be the case that the PP is the crucial piece of information at some point in the discourse, as argued at length in Simons, Tonhauser, Beaver \& Roberts 2011. For instance, with (6), if the speaker happens to base an argument on the existence of a sister and if some other participant (i) happens to believe that the speaker might be lying and (ii) considers the argument as consequential to a sufficient extent, she might attack the PP to defeat the argument. Alternatively, if nothing essential hinges on the fact that the speaker has a sister or that, actually, not everybody was aware of the speaker having a sister, (6) can perfectly well go on its way without further ado.

Summarizing, the fate of (6) in the subsequent part of the conversation turns on factors which are essentially the same for the MC and the PP. This does not entail that the initial situation, which obtains just after the utterance has been issued, is the same for the MC and the PP. In order to preserve Stalnaker's central insight and to avoid the interpretation problems noted for (6), we formulate a more cautious definition of (speaker) PP.

(8) A discourse participant $a$ attributes to a speaker $s$ issuing an utterance $u$ the 
presupposition that $\phi$ if and only $a$, after hearing $u$, believes that $s$ acts as if she believes that $\phi$ is in the common ground or will be in the common ground after $u$, where the common ground is the set of accepted beliefs, as in (5).

There are mainly two scenarios of attribution. Either $\phi$ is a manifest piece of information, which is presumably accessible to everybody, or $s$ uses a form of words through which she communicates that she presupposes that $\phi$. The latter case corresponds to the use of a PP trigger.

A PP trigger in an utterance $u$ is a form of words by which a speaker conventionally communicates that she believes that the presupposed part is in the common ground or will be in the common ground after $u$.

The conventional character of PP triggers is in line with Stalnaker's own perspective, see for instance Stalnaker 2002: 705. However, the definition is restricted to linguistic or communicative PPs and does not cover cases of purely pragmatic PPs, those which concern accessible information in the context without any linguistic/signaling correlate.

Gathering the three features we have reviewed, paired information, discourse non-integration and backgroundedness, we see that, in spite of their differences, they converge towards the idea of an asymmetry between MC and PP, more precisely the idea that, unless the context gives evidence to the contrary, the PPs are subtracted from the main flow of discourse. They are 'normally' not going to be confirmed, rejected or discussed in any way, even though participants in the conversation can decide otherwise (conversational planning) in function of the context.

\subsection{Topic, focus and salience}

Under the perspective of Section 2.1, the MC vs. PP asymmetry closely parallels the focus vs. topic asymmetry and might prove to be essentially similar. More precisely, adopting Erteschik-Shir's (2007) framework, we assume that topics and focuses can be represented as a dynamic structure of pointers (discourse referents) with a recency hierarchy. The basic idea is that recovering a topic entails finding a recent pointer and introducing a focus entails creating a new pointer and assigning high recency to it (indefinites) or locating an existing pointer and assigning high recency to it (definites). As usual, pointers may have different types, referring for instance to individuals or events.

For instance, consider (10). One can distinguish two focuses, F1 and F2. F1 corresponds to a question of the form Why $p$, where $p$ is the proposition that John stopped smoking. $p$ is a PP of (10-A). F2 correspond to the property of being too expensive, which is asserted of the topic it, co-referential with the PP $p^{\prime}$ of $p$, that 
Presuppositions and salience

John had been smoking. So we have two PPs, each one providing a topic for a focus in (10-B).

(10) A: Why did John stop smoking?

$\mathrm{B}$ : [Because $[\mathrm{it}]_{\mathrm{T} 2}$ [was too expensive. $\left.]_{\mathrm{F} 2}\right]_{\mathrm{F} 1}$.

While (10) is a natural exchange, (11) sounds odd. The reason for this oddness is intuitively clear: we are trying to force a topic-source element (the cake) into a typical focus slot (a cleft NP).

(11) A: Who ate the cake, John or Mary?

B: \# It was the cake that John ate.

It has been shown in Cowles, Kluender, Kutas \& Polinsky 2007 that dialogs like (11) elicit specific brain responses. The main goal of Cowles et al.'s (2007) experiment is to compare the on-line reactions of participants to congruent and incongruent dialogs like the following one.

(12) A: A queen, an advisor and a banker were arguing over taxes. Who did the queen silence with a word, the banker or the advisor?

B: It was the banker that the queen silenced. CONGRUENT

C: It was the queen that silenced the banker. INCONGRUENT

Cowles et al. used the standard technique of measuring voltage changes on the scalp by placing electrodes on it and amplifying the signal. Plotting the variations over time gives an electroencephalogram (EEG), from which one can extract particular waveforms or potentials associated with specific events (bodily movements, peceptions, cognitive tasks, etc.), hence the name event-related potential (ERP). ERPs are classified by their orientation, (average) latency and scalp localization. For instance the code $N 400$ refers to a negative deflection peaking somewhere between 200 and $500 \mathrm{~ms}$ after stimulus onset for visual presentation of words. It is generally maximal at centro-parietal electrodes and tends to be left-lateralized for visual presentation of words. The zoo of ERPs is rich (Kappenman \& Luck 2011) but the three main ERPs of interest for linguists are the P300, the N400 and the $\mathrm{P} 600$. The P300 is usually associated with attentional phenomenal and is not directly relevant in the context of our experiments. The N400 is taken to be, among other things, an indicator of semantic incompatibility or discourse non-plausibility (Kutas \& Federmeier 2011). The status of the P600 is less clear. It has been observed for syntactic reprocessing or conflicts but recent work suggests that it has a wider range of causes, having to do with integration difficulties in information processing (Brouwer, Fitz \& Hoeks 2012). 
The main finding ${ }^{3}$ in Cowles et al. 2007 is the presence of a negative deflection between 200 and $500 \mathrm{~ms}$ after the critical word onset (banker vs. queen in (12-B) vs. (12-C)). Cowles et al. (2007) discuss the morphology of the observed waveform and argue that it should be considered as a member of the N400 family.

If the mentioned N400 indexes essentially the mismatch between plausible and actual focus, as in (11) and (12), one is tempted to predict that examples like (10) should not elicit the same brain response. However, on closer look, (10) reveals a peculiar structure. The MC of (10-B) can be paraphrased by "The reason why John stopped smoking is that smoking was too expensive". The it topic in (10-B) is involved into a proposition (that smoking was too expensive) which is part of the focus (F1) of the answer to the (10-A) question. The representation in (10-B) suggests that there is no topic paired with the F1 focus. An alternative approach, in the spirit of QUD-based theories, would posit a topic corresponding to the proposition that John stopped smoking. The form of (10-B) would then be (13).

(13) [John stopped smoking $]_{\mathrm{T} 1}$ [because $[\mathrm{it}]_{\mathrm{T} 2}$ [was too expensive. $\left.]_{\mathrm{F} 2}\right]_{\mathrm{F} 1}$.

The focus of (13) contains a reference (T2) to the PP of the topic (T1) but it is connected to the MC of the same topic. F1 is an explanation of why T1 holds, not of why John had been smoking (the PP of T1). In this respect, it complies with the linking law of Ducrot $1972^{4}$, that is, the constraint according to which, with certain discourse markers (including causal/explanatory ones) it is not possible to attach a discourse constituent to the PP of a different constituent exclusively.

Combining the restrictions on focus congruence illustrated by Cowles et al. 2007 and the linking law of Ducrot 1972 does not give us the whole story. We don't know what happens in general when we select as topic a PP exclusively. In Jayez 2010 it is shown that native speakers massively reject forced attachments to the PP alone, but there are only two types of discourse connections studied in Jayez's work, consequence (therefore type) and justification (because type). Moreover, the experimental procedure is based on naturalness judgments and does not provide any insight on the on-line processing of sentences. Given the intuition emerging from Section 2.1, it is possible that there is some difference between MC and PP, even though there is no blatant violation of focus congruence or the linking law. If the MC is more salient or activated than the PP, referring to the PP would be less expected than referring to the $\mathrm{MC}$ and a sentence that would require the nonpreferred reference in order to be interpreted could be more marked than a sentence compatible with the preferred reference. This markedness difference might then have an effect on the cognitive processing of these two categories of sentences.

3 There are other interesting observations and questions in their work, but they seem to be orthogonal to our main issue.

4 Loi d'enchaînement in French. 
Presuppositions and salience

Building an experimental strategy to address this possibility has to take into account at least three additional aspects. First, candidate measures for the problem must be time-locked, that is, they must follow the real processing sequence as tightly as possible. Second, one should compare the MC and the PP for the same trigger. Although comparing the on-line processing of different triggers can provide useful information as to the processing speed (Schwarz 2015), it is more difficult to draw conclusions than in the unique trigger configuration. Third, the variety of triggers creates possible confounds. With aspectual verbs such as start or stop, the MC coincides with the final stage of the transition. Several experimental studies suggest that there is a cognitive advantage for the endpoint of a transition. For instance, Nuthmann \& van der Meer (2005) observe a stronger pupillary response and longer response times for pairs like shrinking-small than for pairs like shrinking-large, which suggests that the final stage is cognitively dominant in the representation of transitions. If this is true, this feature could seriously bias a study comparing the MC and the PP of aspectual verbs, since the MC could inherit some cognitive priority from its final status in the transition. Another problematic class is that of exclusives, whose semantic status is complex (Coppock \& Beaver 2014). Even if we adopt a conservative stance and assume that exclusives presuppose a proposition corresponding to the modified sentence (e.g. only $\mathrm{S}$ presupposes the proposition expressed by $\mathrm{S}$ ), the fact remains that the $\mathrm{PP}$ is explicit whereas the $\mathrm{MC}$ is not. This difference in the degree of explicitness might influence the processing in a way orthogonal to our primary concern. Finally, anaphoric elements like too involve a number of parameters in addition to the MC vs. PP distinction, in particular distance to antecedent (Callahan 2009; Kim 2015).

Given these potential sources of difficulty, we decided to start with factive verbs, because they seem to be free from possibly interacting factors and provide the nearest equivalent to a baseline with respect to explicitness, anaphoric distance and temporal ordering. The idea was to compare reference to the MC and the PP after a factive sentence. Following Winterstein 2011, we used an additive discourse relation marked by the French adverb aussi (too, also), in order to circumvent the strong effect of consequence and justification discourse relations (Ducrot 1972; Jayez 2010). The two basic patterns are shown in (14) and (15). (14) uses the masculine clitic pronoun le to refer either to the proposition corresponding to the complement clause of the first sentence (Peter takes the bus) or to the complement noun (the bus). Coreference is indicated by underlining. In (14), at the moment the clitic is seen, it is ambiguous between a propositional and an individual denotation. In (15), the whole complement sentence or noun is repeated and the ambiguity does not extend beyond the additive adverb (aussi).

a. Paul sait que Pierre prend le bus. Louis aussi le sait. Paul knows that Peter takes the bus. Louis too it knows 
'Paul knows that Peter takes the bus. Louis too knows that.'

b. Paul sait que Pierre prend le bus. Louis aussi le prend.

Paul knows that Peter takes the bus. Louis too it takes

'Paul knows that Peter takes the bus. Louis too takes it.'

a. Paul sait que Pierre prend le bus. Louis aussi sait que Pierre

Paul knows that Peter takes the bus. Louis too knows that Peter

prend le bus;

takes the bus

'Paul knows that Peter takes the bus. Louis too knows that Peter takes the bus.'

b. Paul sait que Pierre prend le bus. Louis aussi prend le bus.

Paul knows that Peter takes the bus. Louis too takes the bus

'Paul knows that Peter takes the bus. Louis too takes the bus.'

In order to tap into the on-line processing of the sentence, we ran two EEG experiments on the clitic and non-clitic patterns. If reference to the PP is dispreferred, we expect to detect an identifiable ERP (typically, an 400 or a P600) shortly after the onset of the word that dissipates the referential ambiguity, as indicated by the arrow in (16) for the case with a clitic.

(16) a. Paul sait que Pierre prend le bus. Louis aussi le sait $\uparrow$ Paul knows that Peter takes the bus. Louis too it knows

b. Paul sait que Pierre prend le bus. Louis aussi le prend $\uparrow$ Paul knows that Peter takes the bus. Louis too it takes

\section{The experiments}

\subsection{Experiment 1 (the clitic pattern)}

Participants 31 undergraduate students were recruited. All were right-handed native speakers without neurological disorders and with normal or corrected to normal vision. They were paid for their participation. After artifact rejection, 9 participants were not kept for data analysis. Of the remaining 22 participants, 14 were female and 8 male with an age range of 18-24 for a mean of 20.7.

Design and materials We used 132 sentences, 88 sentences based on 22 factive verbs plus 44 fillers. The 88 experimental sentences were divided into two groups of 44 sentences each, corresponding to the reference to the MC vs. reference to the PP conditions. Each factive verb appeared two times under each condition. 
Presuppositions and salience

The frequencies, syllable number and letter number of MC and PP verbs were aligned and morphological/phonological competitors were avoided. We used 132 first-name triples, so that the first-names were different for each stimulus and no first-name was repeated across stimuli. About $25 \%$ of the sentences were followed by a comprehension yes-no question to keep participants attentive. 12 questions concerned the fillers and 24 the experimental stimuli. The stimuli were pseudorandomized so that each set of stimuli started with a filler and there was a regular pattern, each filler being followed by two experimental stimuli. The disambiguating verb was always the last word of the sentence.

Measures Electro-physiological data were recorded from a 128 channel dense array EEG using an EGI ${ }^{\mathrm{TM}}$ Geodesic Sensor Net with standard settings for language processing (vertex $\mathrm{Cz}$ electrode as online reference, epochs between -300 and 1000 ms around onset, sampling rate of $500 \mathrm{~Hz}^{5}$, baseline correction of $300 \mathrm{~ms}$ ). Artifacts were semi-automatically rejected with BrainVision Analyzer 2. Due to a noise percentage of $25 \%$ or higher, 9 participants were taken out.

Procedure Participants were seated in a quiet room in front of a computer screen. They were instructed to read silently the contents of the screen. The full first sentence appeared on the screen. After they had understood the first sentence, they pressed a key and the second sentence appeared word by word in the center of the screen, following the Rapid Serial Word Paradigm with 300 ms duration and 300 ms blank screen (SOA $600 \mathrm{~ms}$ ). They answered the yes-no questions by pressing dedicated colored keys on the keyboard of the computer. A five-minute break occurred in the middle of the experiment (66 stimuli).

Results The electrode array was divided into a number of regions, frontal, temporal, central, parietal, fronto-central, fronto-temporal and centro-parietal, which have been shown to host interesting scalp voltage differences in various experimental procedures. For a number of temporal windows, a one-way analysis of variance with repeated measures was conducted for each region in order to compare the two conditions of reference to the MC vs. reference to the PP. A parallel linear mixed-model analysis with intercept and slope correction for participants and electrodes as random factor was conducted. The waveforms for the different sites are shown in Figure 1 on page 613.6 The $250-550$ time window does not suggest any N400 effect and the

5 So a data point corresponds to $2 \mathrm{~ms}$ in the signal.

6 The extended electrode sites correspond to a slightly larger number of electrodes for some standard regions. 
differences between $\mathrm{MC}$ and $\mathrm{PP}$ are always non-significant for all regions. However the time region of the $\mathrm{P} 600$ gives rise to a positive deflection at fronto-central electrodes and centro-parietal electrodes seem to show a late positivity. The differences are significant in each case: $F(1,18)=9.6, p=0.006, t=-2.255$ (reference level $=\mathrm{MC}$ ) for the fronto-central electrodes in the 550-800 range, $F(1,18)=11.8$, $p=0.003, t=-3.1$ for the extended fronto-central electrodes in the 550-800 range, $F(1,18)=9.8, p=0.0057, t=-2.5$ for the centro-parietal site in the $650-900$ range, $F(1,18)=10.43, p=0.0046, t=-2.78$ for the extended centro-parietal site in the 650-900 range.

However, two aspects have to be taken into account. The amplitude of the positive deflection is low. We are around 2 and, so, far from the 4-5 amplitudes generally reported in papers mentioning a P600 or a late positivity. Moreover, the clitic used in the experiment might trigger an effect that we did not control. In one case it must resolved to a nominal complement denoting a physical object (the bus). In the other case it is resolved to a clause denoting an abstract object (a proposition or a fact). We don't know whether these differences are marginal or have a noticeable impact on the processing. This question motivated the construction of another experiment without the clitic.

\subsection{Experiment 2 (the non-clitic pattern)}

Participants Thirty undergraduate students were recruited. All were right-handed native speakers without neurological disorders and with normal or corrected to normal vision. They had not participated in experiment 1 and were paid for their participation. After artifact rejection, 9 participants were not kept for data analysis. Of the remaining 21 participants, 10 were female and 11 male with an age range of 18-27 for a mean of 20.9 .

Design, materials and procedure The stimuli in experiment 2 were absolutely parallel to those of experiment 1 (the same verbs occurring with the same first names and complement clauses/nouns in the same order). The only difference was that the clitic was replaced by the full complement, as in (15). The procedure was the same as in experiment 1 .

Results The visual results are in Figure 2 on page 614. The data were subjected to the same analysis as for experiment 1 The P600-like effect on the MC disappeared completely at centro-parietal and fronto-central sites. There are two other candidates for a difference. At fronto-central site, the PP is visually higher in the 650-900 window, but the difference is not significant $(F(1,17)=3.045, p=0.1$, 
Presuppositions and salience

$t=1.58$ with reference-level on the $\mathrm{MC}$, for the extended fronto-central set of electrodes). At frontal and fronto-central sites the MC is visually lower in the 250-400 window. For frontal and fronto-central electrodes, the difference does not reach significance $F(1,17)=3.415, p=0.08, t=1.713$ with reference-level on the MC, and $F(1,17)=4.057, p=0.06, t=1.752$. It is significant for extended fronto-central site: $F(1,17)=5.023, p=0.04, t=2.09$.

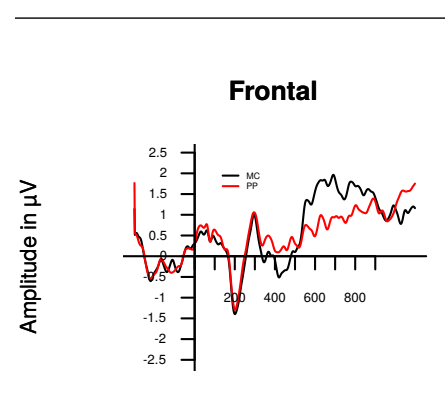

Time in ms

Central

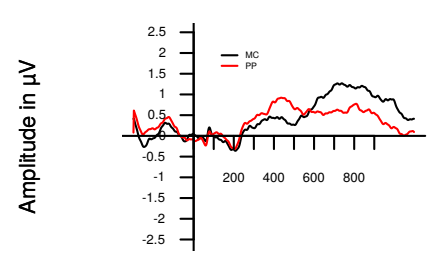

Time in ms

Fronto-Central

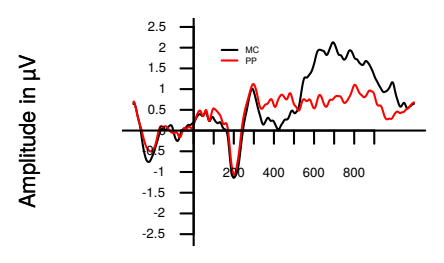

Time in $\mathrm{ms}$

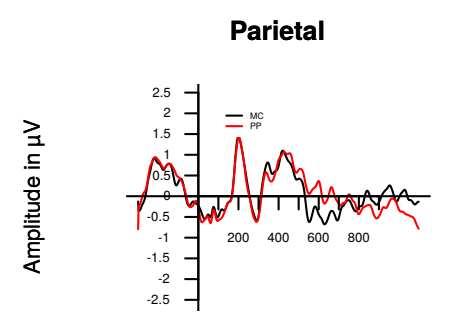

Time in ms

Centro-Parietal

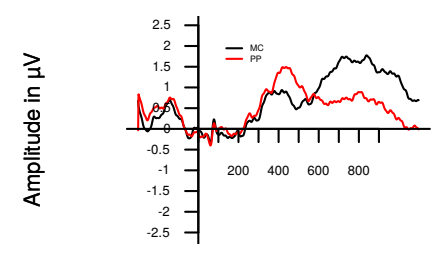

Time in ms

Fronto-Central (extended)

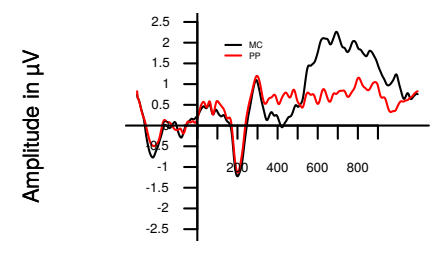

Time in $\mathrm{ms}$

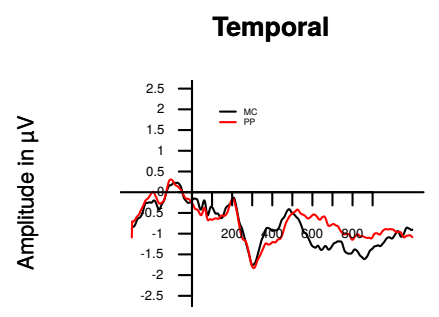

Time in ms

Centro-Parietal (extended)

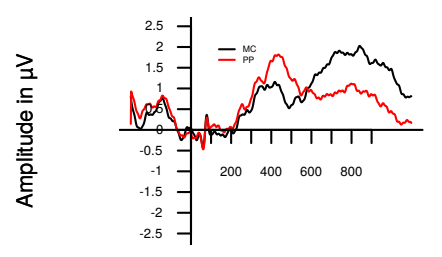

Time in ms

Fronto-Temporal

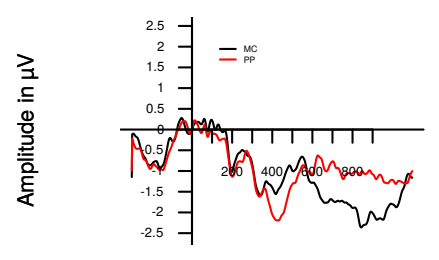

Time in ms

Figure $1 \quad \mathrm{MC}$ and PP waveforms for experiment 1 


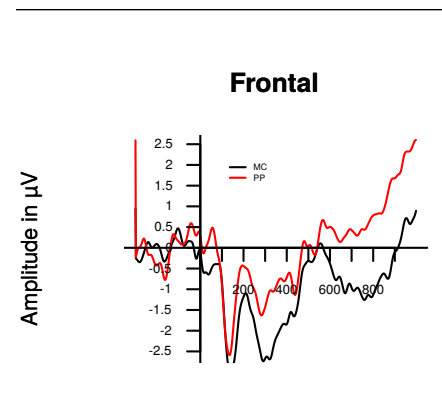

Time in $\mathrm{ms}$

Central

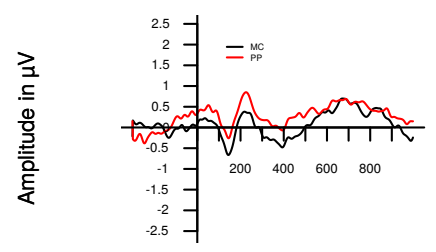

Time in ms

Fronto-Central

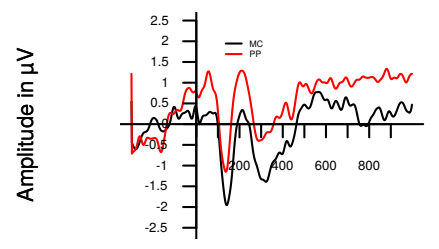

Time in ms

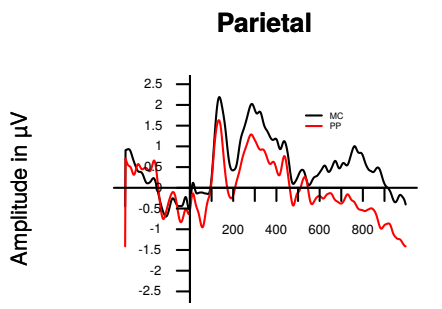

Time in ms

Centro-Parietal

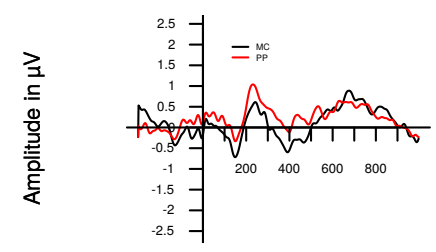

Time in $\mathrm{ms}$

Fronto-Central (extended)

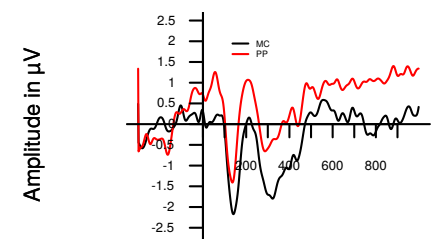

Time in $\mathrm{ms}$

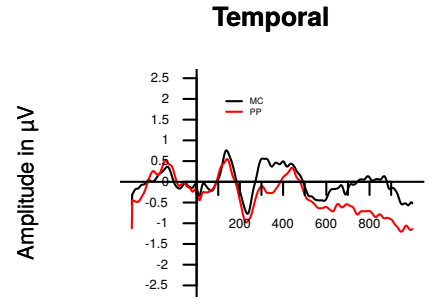

Time in ms

Centro-Parietal (extended)

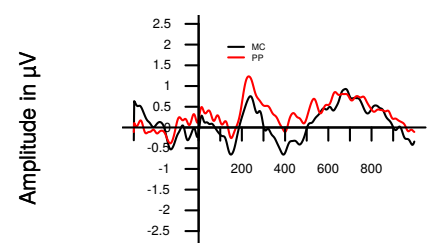

Time in $\mathrm{ms}$

Fronto-Temporal

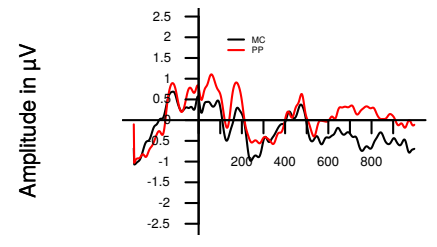

Time in $\mathrm{ms}$

Figure $2 \quad \mathrm{MC}$ and $\mathrm{PP}$ waveforms for experiment 2

\subsection{Discussion}

The most obvious aspect of the results is the absence of any strong effect (amplitude waveform $\gtrsim 4)$. In particular, there is no evidence of an N400 or P600 effect, once one gets rid of the clitic. This is compatible with the following hypothesis: the PP of factive verbs is as accessible as the MC when it forms the topic of an additive relation marked by aussi (too). It is also in agreement with Winterstein's (2011) 
claim that aussi can freely refer to a PP whenever it plausible, given the context and the world-knowledge. More precisely, the present results suggest the following disjunction: (i) there is no salience difference between the MC and the PP of factive verbs or (ii) such a difference exists but does not affect the referring preferences of aussi, whether in terms of deviance (N400) or in terms of reprocessing (P600). The empirical support for this disjunction comes from the fact that, when we observe differences, their amplitude is much more limited than what is described in the ERP literature, and that the most significant difference is observed for the experiment with clitic in the centro-parietal and fronto-central regions and is absent in the experiment without clitic, which suggests that it is the clitic pronoun and not the MC vs. PP contrast which is responsible for the difference in waveforms. This is not surprising if one keeps in mind that, in contrast to the experiment without clitic, the clitic pronoun has to be resolved in experiment 1 and this is likely to interact with the constraints affecting anaphora resolution.

Concerning the 250-400 region, we noticed a more negative deflection for the $\mathrm{MC}$ at the frontal and fronto-central sites. Although the size of the deflection is small (ミ-2.5), the tendency seems to be robust at these sites. In the classification of ERPs, what we observed can be tentatively assimilated to an N300. The N300 ERP is usually observed in the context of visuo-spatial processing (e.g. repetition in face presentation) or emotional particles (exclamations). Franklin, Dien, Neely \& Huber (2007: 1065) speculate that the N300 effect they observed in a priming task "reflects an expectancy process that is sensitive to category membership and/or semantic similarity". A replication of experiment 2 is necessary to make sure that the observed waveform is not accidental. If it is not, it can be taken as an indication of a difference between MC and PP.

The interpretation is complicated by the possibility that the observed waveform just mirrors the parietal waveforms, as suggested by the first two waveforms of Figure 2 and their combined version in Figure 3, in which case we would just observe the mirror image of a relatively expected attention-driven P300. 
Frontal compared to Parietal

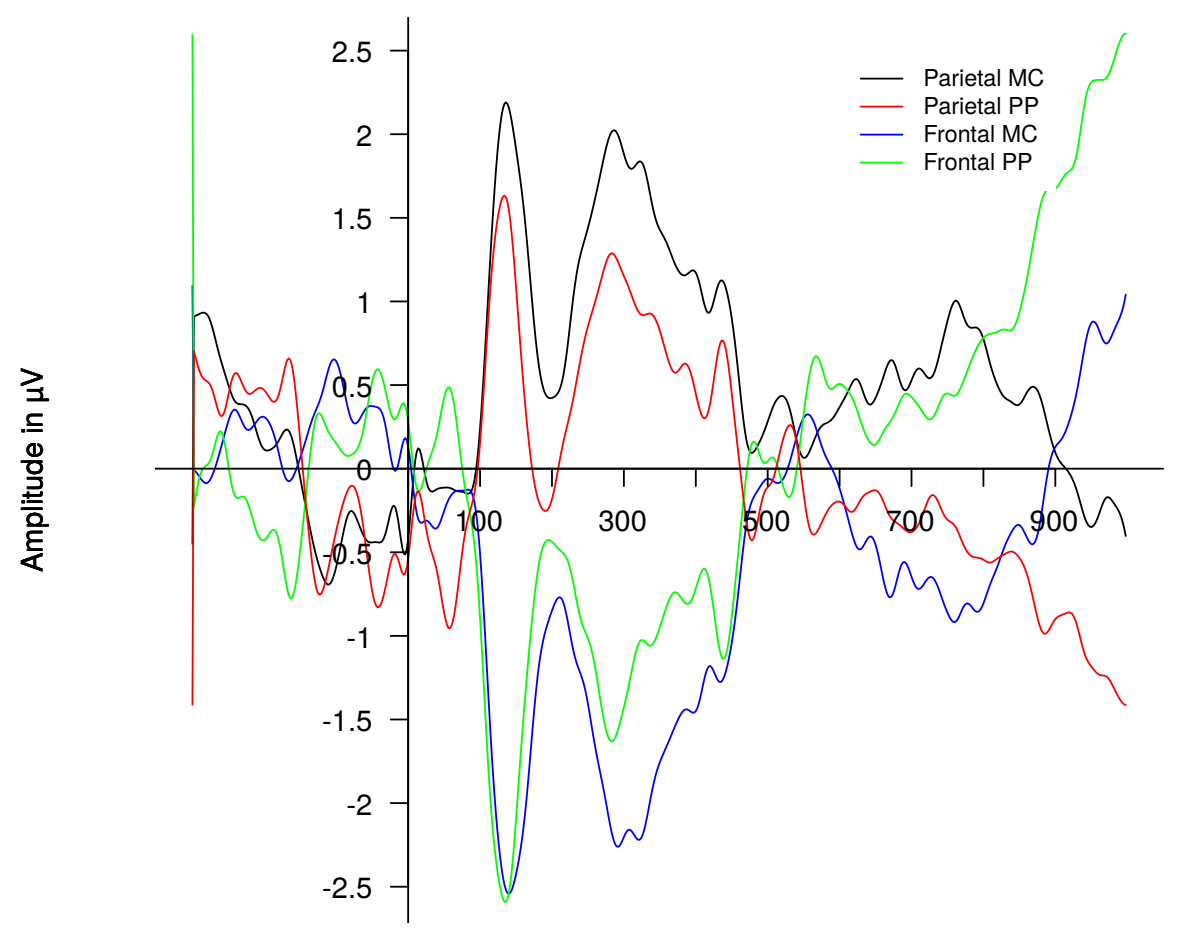

Time in ms

Figure 3 A mirror effect?

\section{Conclusion}

In this paper we have presented the first stage of an investigation of the differential properties of MC and PP from an experimental point of view. We have shown that, for factive verbs, reference to the MC or the PP by means of aussi (too) does not give rise to an N400 or P600 ERP. We have noticed a potential N300 difference, which remains to be confirmed and interpreted. The design of the two reported experiments makes the $\mathrm{PP}$ or the $\mathrm{MC}$ a topic and the discourse relation an additive one. It is thus legitimate to ask whether one would obtain similar results when the $\mathrm{MC} / \mathrm{PP}$ is a focus and/or the discourse relation is different. The answer to the topic/focus part of the questions seems obvious. Given the projection properties and the non-answerhood of PPs (Grimshaw 1979), we expect to see brain signals of 
Presuppositions and salience

deviation. However, the nature of these signals (for instance, N400, P600 or some combination thereof) remains to be established. The combination of topic status with non-additive discourse relations for factive verbs is the next step on our experimental agenda.

\section{References}

Abbott, Barbara. 2008. Presuppositions and common ground. Linguistics and Philosophy 21. 523-538. doi:10.1007/s10988-008-9048-8.

Beaver, David I. \& Bart Geurts. 2013. Presupposition. In Edward N. Zalta (ed.), The Stanford Encyclopedia of Philosophy (Fall 2013 Edition), http://plato.stanford. edu/archives/fall2013/entries/presupposition/.

Brouwer, Harm, Hartmut Fitz \& John Hoeks. 2012. Getting real about semantic illusions: Rethinking the functional role of the p600 in language comprehension. Brain Research 1446. 127-143. doi:10.1016/j.brainres.2012.01.055.

Callahan, Sarah M. 2009. A multi-methodological investigation of the processing and interpretation of coordinate sentences involving verb phrase anaphors: UC San Diego PhD dissertation. http://escholarship.org/uc/item/42g752wv.

Chierchia, Gennaro \& Sally McConnell-Ginet. 1990. Meaning and Grammar: An Introduction to Semantics. Cambridge (MA): MIT Press.

Coppock, Elizabeth \& David Beaver. 2014. Principles of the exclusive muddle. Journal of Semantics 31(3). 371-432.

Cowles, H. Wind, Robert Kluender, Marta Kutas \& Maria Polinsky. 2007. Violations of information structure: An electrophysiological study of answers to wh-questions. Brain and Language 102(2). 228-242. doi:10.1016/j.bandl.2007.04.004.

Ducrot, Oswald. 1972. Dire et ne pas dire. Paris: Hermann.

Erteschik-Shir, Nomi. 2007. Information Structure. The Syntax-Discourse Interface. Oxford Surveys in Syntax and Morphology. New York: Oxford University Press. von Fintel, Kai. 2006. What is presupposition accommodation, again? In Workshop on Presupposition Accommodation, October 13th - October 15th, 2006. Ohio State University.

Franklin, Michael S., Joseph Dien, James H. Neely \& Elizabeth Huber. 2007. Semantic priming modulates the $\mathrm{n} 400, \mathrm{n} 300$, and n400rp. Clinical Neurophysiology 118. 1053-1068. doi:10.1016/j.clinph.2007.01.012.

Geurts, Bart. 1999. Presuppositions and Pronouns. (Current Research in the Semantics/Pragmatics Interface 3). Oxford: Elsevier.

Ginzburg, Jonathan. 2012. The Interactive Stance. Meaning for Conversation. Oxford: Oxford University Press. 
Jayez and Reinecke

Grimshaw, Jane. 1979. Complement selection and the lexicon. Linguistic Inquiry 10(2). 279-326.

Jayez, Jacques. 2010. Projective meaning and attachment. In Logic, Language and Meaning. Revised Selected Papers of the 17th Amsterdam Colloquium, Amsterdam 2009 (Lecture Notes in Artificial Intelligence 6042), 325-334. Berlin: Springer.

Kappenman, Emily S. \& Steven J. Luck (eds.). 2011. The Oxford Handbook of Event-Related Potentials. Oxford Library of Psychology. Oxford, New York: Oxford University Press.

Kim, Christina S. 2015. Presupposition satisfaction, locality and discourse constituency. In Florian Schwarz (ed.), Experimental Perspectives on Presuppositions (Studies in Theoretical Psycholinguistics 45), 109-134. Switzerland: Springer International Publishing.

Kutas, Marta \& Kara D. Federmeier. 2011. Thirty years and counting: Finding meaning in the $\mathrm{n} 400$ component of the event-related brain potential (erp). Annual Review of Psychology 62. 621-647. doi:10.1146/annurev.psych.093008.131123.

Nuthmann, Antje \& Elke van der Meer. 2005. Time's arrow and pupillary response. Psychophysiology 42. 306-317. doi:10.1111/j.1469-8986.2005.00291.x.

Potts, Christopher. 2005. The Logic of Conventional Implicatures. Oxford Studies in Theoretical Linguistics. Oxford: Oxford University Press.

Roberts, Craige. 2012. Information structure in discourse: Towards an integrated formal theory of pragmatics. Semantics and Pragmatics 5(6). 169. doi:10.3765/sp.5.6. This is a reissue of a 1998 version to be found at http://ling.osu.edu/ croberts/infostr.pdf.

Schwarz, Florian. 2015. Presupposition vs. asserted content in online processing. In Florian Schwarz (ed.), Experimental Perspectives on Presuppositions (Studies in Theoretical Psycholinguistics 45), 89-108. Switzerland: Springer International Publishing.

Simons, Mandy, Judith Tonhauser, David Beaver \& Craige Roberts. 2011. What Projects and Why. In Nan Li \& David Lutz (eds.), Semantics and Linguistic Theory (SALT) 20, 309-327. eLanguage.

Stalnaker, Robert. 1974. Pragmatic presuppositions. In Milton K. Munitz \& Peter Unger (eds.), Semantics and Philosophy, 197-214. New York: New York University Press.

Stalnaker, Robert. 2002. Common ground. Linguistics and Philosophy 25. 701-721.

Stalnaker, Robert. 2008. A response to Abbott on presupposition and common ground. Linguistics and Philosophy 31. 539-544. doi:10.1007/s10988-0089047-9.

Szabó, Zoltán Gender. 2006. Comments on Kai von Fintel's paper. In Workshop on Presupposition Accommodation, October 13th - October 15th, 2006. Ohio State 
Presuppositions and salience

University.

Winterstein, Grégoire. 2011. The meaning of the additive too: Presupposition and discourse similarity. In Nick Bezhanishvili, Sebastian Löbner, Kerstin Schwabe \& Luca Spada (eds.), Logic, Language and Computation. 8th International Tbilisi Symposium on Logic, Language, and Computation, TbiLLC 2009. Revised Selected Papers (Lecture Notes in Artificial Intelligence 6618), 322-341. Berlin, Heidelberg: Springer Verlag. doi:10.1007/978-3-642-22303-7.

Wooldridge, Michael. 2000. Reasoning about Rational Agents. Cambridge (MA): MIT Press.

Jacques Jayez

ENS de Lyon, 15 Parvis Descartes BP 700069342 Lyon Cedex 07 France jacques.jayez@ens-lyon.fr ISC Marc Jeannerod, UMR 5304 CNRS 67 Bld Pinel 69675 Bron Cedex

jjayez@isc.cnrs.fr
Robert Reinecke

ISC Marc Jeannerod, UMR 5304 CNRS 67 Bld Pinel 69675 Bron Cedex rreineck@isc.cnrs.fr 